(C) 2006 The Japan Society of Applied Physics

\title{
Resolving Nano Scale Recording Bits on Phase-Change Rewritable Optical Disk
}

\author{
Shih Kai LIN ${ }^{1,2}$, Peilin YANG ${ }^{1,2}$, I Chun $\operatorname{LIN}^{1,2}$, Hao Wen HsU ${ }^{1}$ and Din Ping TSAI ${ }^{1,2 *}$ \\ ${ }^{1}$ Center of Nanostorage Research, National Taiwan University, Taipei 10617, Taiwan \\ ${ }^{2}$ Department of Physics, National Taiwan University, Taipei 10617, Taiwan
}

(Received August 18, 2005; accepted November 25, 2005; published online February 24, 2006)

We use a new imaging method to resolve the nano scale recording bits on phase-change rewritable optical disks. By this method, several kinds of nano scale recording bits on different phase-change optical disks can be clearly imaged without any painstaking or expensive procedures. With the conductive-atomic force microscopy (C-AFM) images of nano scale recording bits on phase-change materials, we can study the properties of phase-change recording layers and the recording bit formation mechanism comprehensively. [DOI: 10.1143/JJAP.45.1431]

KEYWORDS: conductive-atomic force microscopy (C-AFM), rewritable optical disk, digital versatile disk (DVD), phase-change material, recording bit, Blu-ray disk

\section{Introduction}

The digital versatile disk (DVD) has been an indispensable multimedia and data storage technology for decades. However, the thorough study of phase-change rewritable optical disks, ${ }^{1)}$ such as DVD rewritable (DVD $\pm \mathrm{RW}$ ), is still incomplete due to the deficiency of infallible and sophisticated methods. The difficulty in observing the recording bits with high spatial resolution and distinction inhibited the further understanding of the key issues in early studies. To accomplish this purpose, recently, several techniques, including scanning near-field optical microscopy (SNOM), ${ }^{2}$ scanning surface potential microscopy (SSPM), ${ }^{3)}$ lateral force microscopy (LFM), ${ }^{4}$ scanning electron microscopy $(\mathrm{SEM}),{ }^{5)}$ and transmission electron microscopy (TEM), ${ }^{6}$ have been applied to obtain pellucid images of nano scale recording bits on phase-change rewritable optical disks. Nevertheless, either the results have never been satisfying, or the processes are often painstaking and inaccessible. So far, the most reliable way of resolving nano scale recording bits on phase-change rewritable optical disks is to take advantage of SEM, or TEM, but the cost is extremely high. Therefore, we tend to make use of a credible and deliberate, but not cumbersome, method for resolving nano scale recording bits in a phase-change recording layer. In the method conductive-atomic force microscopy (C-AFM) is introduced to discriminate the amorphous recording marks from the crystalline region formed by initialization. In this way, the nature of the phase-change recording layer and the characteristics of recording bits, such as shape and length, and the writing strategies can be thoroughly investigated. We are also able to measure recording bits of the nextgeneration large-capacity optical disks: the Blu-ray disk, ${ }^{7)}$ and the near-field optical disk. ${ }^{8-12)}$ In fact, the optical readout signals and the spectrum of carrier-to-noise ratio (CNR) have long been used to gain more laconic and straightforward measurements of recording bits on the optical disks in order to test the performance of optical disks and disk drivers. It is, however, more helpful to obtain real images of nano scale recording bits because then we can exhaustively study both the phase-change rewritable optical disks and disk drivers in connection with writing strategy by arranging serial elaborate recording bits, especially for a recording bit

*E-mail address: dptsai@phys.ntu.edu.tw smaller than the diffraction limit. We also demonstrate actual images of nano scale recording bits on $\mathrm{DVD} \pm \mathrm{RW}$ optical disks as well as on Blu-ray optical disks.

\section{Experimental Setup}

The samples of nano scale recording bits on phase-change rewritable optical disks in this work were prepared mainly using a dynamic optical disk tester ${ }^{13)}$ (DDU-1000, Pulstec, Japan) with a red laser beam $(\lambda=650 \mathrm{~nm})$ and numerical aperture (NA) of 0.65 . We also prepared a sample of recording bits on commercial rewritable optical disks using a static optical tester composed of a commercial polarizedlight microscope with two semiconductor laser diodes operating at $\lambda_{1}=633 \mathrm{~nm}$ (read), and $\lambda_{2}=658 \mathrm{~nm}$ (write). The illustration of our experiment for scanning nano scale recording bits on commercial phase-change optical disks, including a Blu-ray optical disk, is shown in Fig. 1. The most crucial procedure in our experiment is to remove the dielectric layer covering above the phase-change recording layer, so as to achieve good conductivity in the phase-change recording layer. It is constructive that the commercial $\mathrm{DVD} \pm \mathrm{RW}$ optical disk itself is bonded by two polycarbonate substrates so that we can readily withdraw the reflection layer covering the printed side of the polycarbonate substrate and retain the phase-change recording thin film because of the weaker surface attractive force between the phasechange layer and the dielectric layer. Right after the

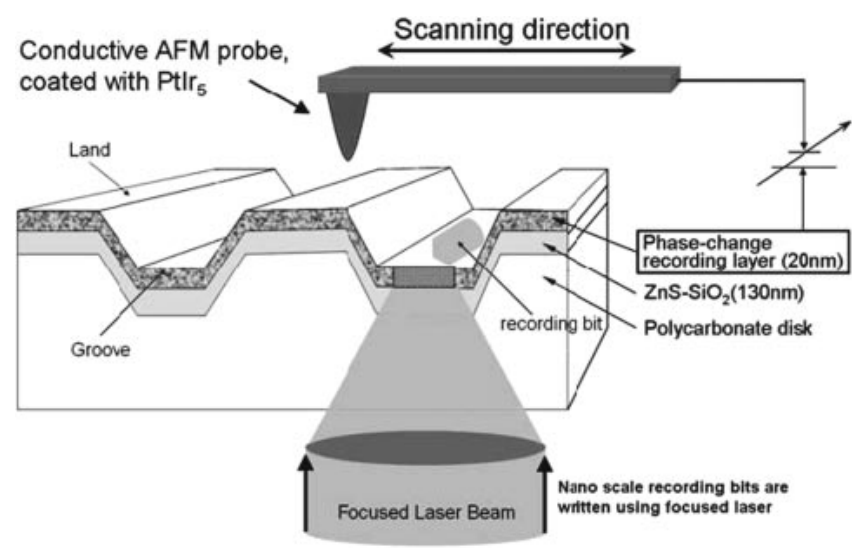

Fig. 1. Schematic diagram of the scanning probe and recording bits on optical disk samples. 


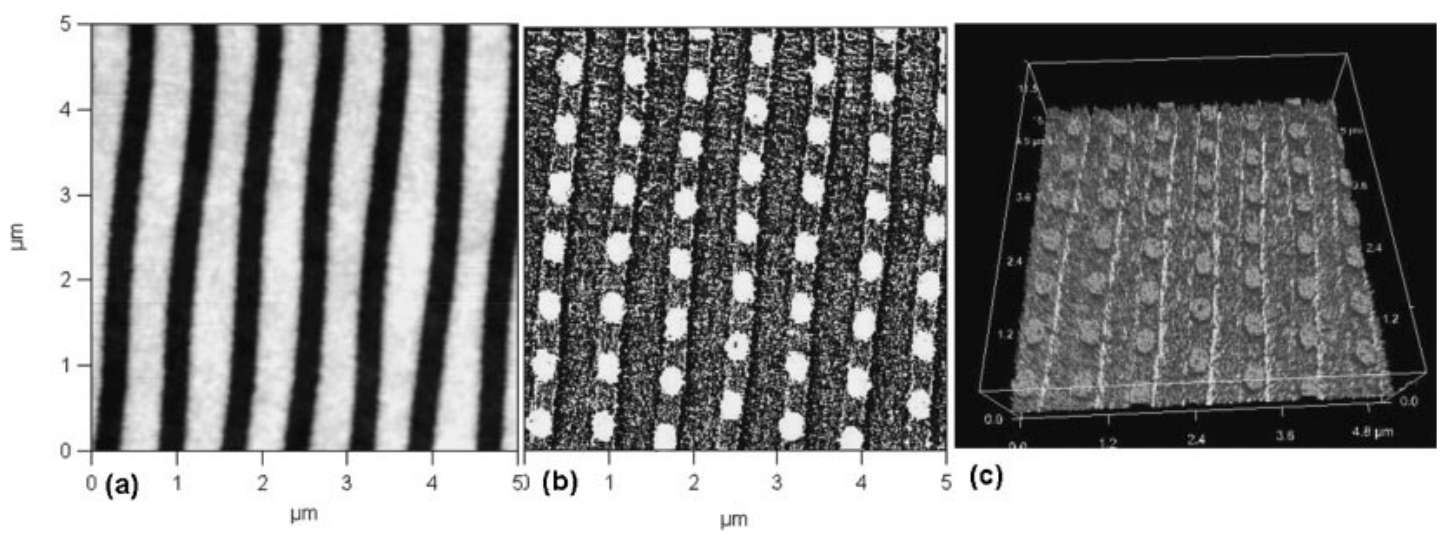

Fig. 2. (a) A $5 \times 5 \mu \mathrm{m}^{2}$ AFM image of recording layer on commercial $4 \times$ DVD + RW optical disk. (b) C-AFM current intensity image of recording bits written using an optical disk tester (DDU-1000). (c) three-dimensional CAFM image of (b).
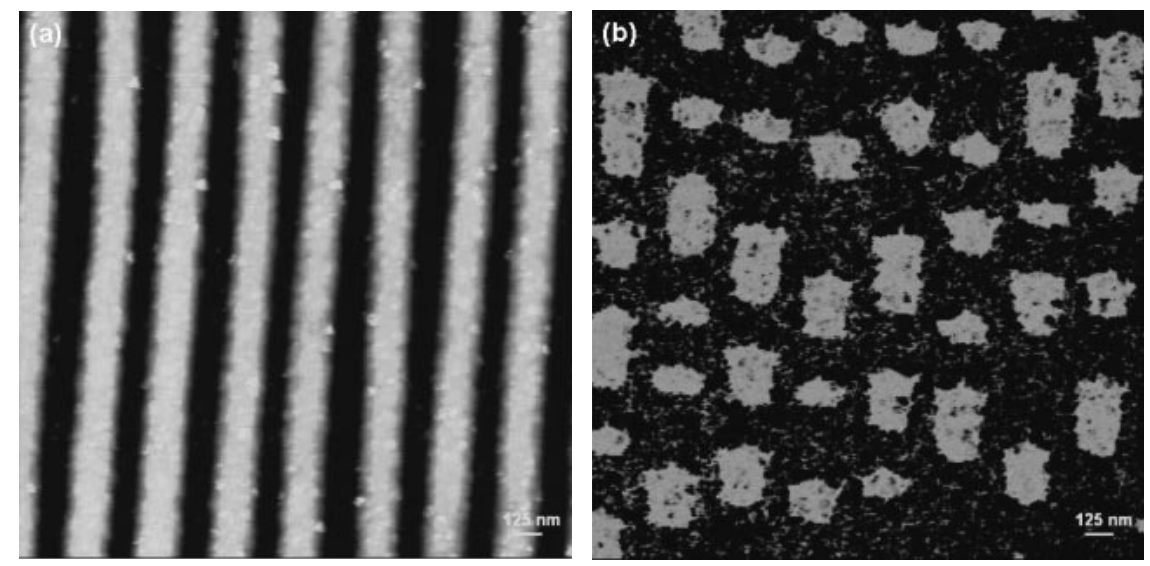

Fig. 3. (a) A $2.5 \times 2.5 \mu \mathrm{m}^{2}$ AFM image of nano scale recording layer on Blu-ray optical disk. (b) C-AFM current intensity image of nano scale recording bits on the same area as shown in (a).

separation from the dielectric layer, the scanning probe of CAFM can be brought into contact with the surface of the phase-change recording layer so as to measure the current densities in a specific local region of the phase-change recording layer. In order to avoid the degradation and contamination of the phase-change recording layer, the measurement should be carried out within one day. The experimental cantilever probes of C-AFM are coated with PtIr5. Images of nano scale recording bits on phase-change optical disks were obtained at fresh regions to eliminate possible artifacts created by the previous scan. We obtained high-quality images by scanning at a constant velocity of $10 \mu \mathrm{m} / \mathrm{s}$ in the contact mode with the proper set point of contact force. All the samples, including the sample of nano scale recording bits on a Blu-ray optical disk, were measured by the same method. The Blu-ray optical disks were commercial available from SONY. and the phase-change rewritable optical disks used in our experiments were made by Ritek Incorporation.

\section{Results and Discussion}

Figure 2 shows the $5 \times 5 \mu \mathrm{m}^{2}$ topographic image as well as the C-AFM image of recording bits on a commercial phase-change rewritable optical disk, and in Fig. 2(a), the darker tracks are the grooves where the recording bits are written. The average height difference between lands and grooves is measured to be about $26 \mathrm{~nm}$. Figure 2(b) shows a clear C-AFM current intensity image of recording bits obtained simultaneously with Fig. 2(a). Figure 2(c) shows a vivid three-dimensional CAFM image of the nano scale recording bits in Fig. 2(b). Because the phase-change recording layer of all commercial DVD+RWs is originally in the crystalline state, the bright areas shown in the C-AFM images of Figs. 2(b) and 2(c) clearly depict the shape of each recording bit, i.e. the area in the amorphous state of the phase-change material. In Fig. 2(b) the accurate information of the size and shape of recording bits on the phase-change recording layer provides us with an advantage in the understanding of the optical and thermal interactions between the phase-change recording materials and the laser input. Figure 3 shows the AFM and C-AFM images of nano scale recording bits on the Blu-ray optical disk, for the scanning area of $2.5 \times 2.5 \mu \mathrm{m}^{2}$. The mark size of each recording bit is random and the smallest length we measured in Fig. 3 is about $120 \mathrm{~nm}$. This clear measurement shows the potential of this method for studying the next-generation optical storage medium of Blu-ray disks. Figure 4(a) shows a micrograph of recording marks on a phase-change commercial $\mathrm{DVD} \pm \mathrm{RW}$ optical disk; those recording marks were written using a static tester with $15 \mathrm{~mW}$ writing power. Figures 4(b) and 4(c) show the AFM and C-AFM images of the recording marks displayed in Fig. 4(a). With the 


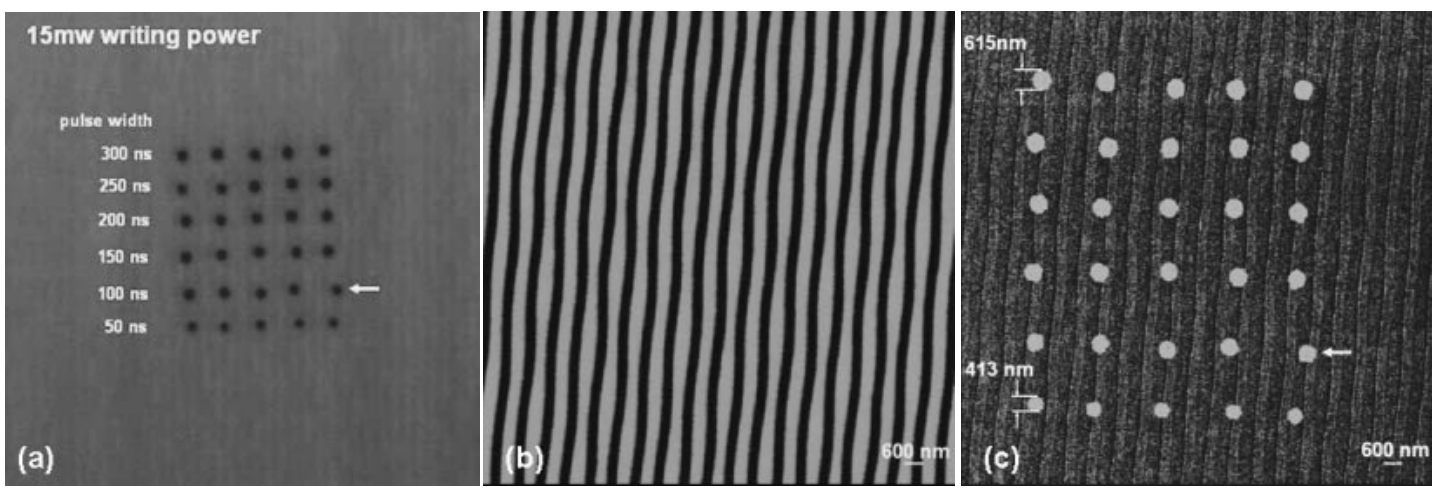

Fig. 4. (a) CCD micrograph of recording marks written using static tester on commercial phase-change rewritable optical disk. The center-to-center spacing between contiguous marks is $2 \mu \mathrm{m}$. (b) A $15 \times 15 \mu \mathrm{m}^{2} \mathrm{AFM}$ image of recording layer on commercial phase-change rewritable optical disk. (c) C-AFM current intensity image of recording marks.

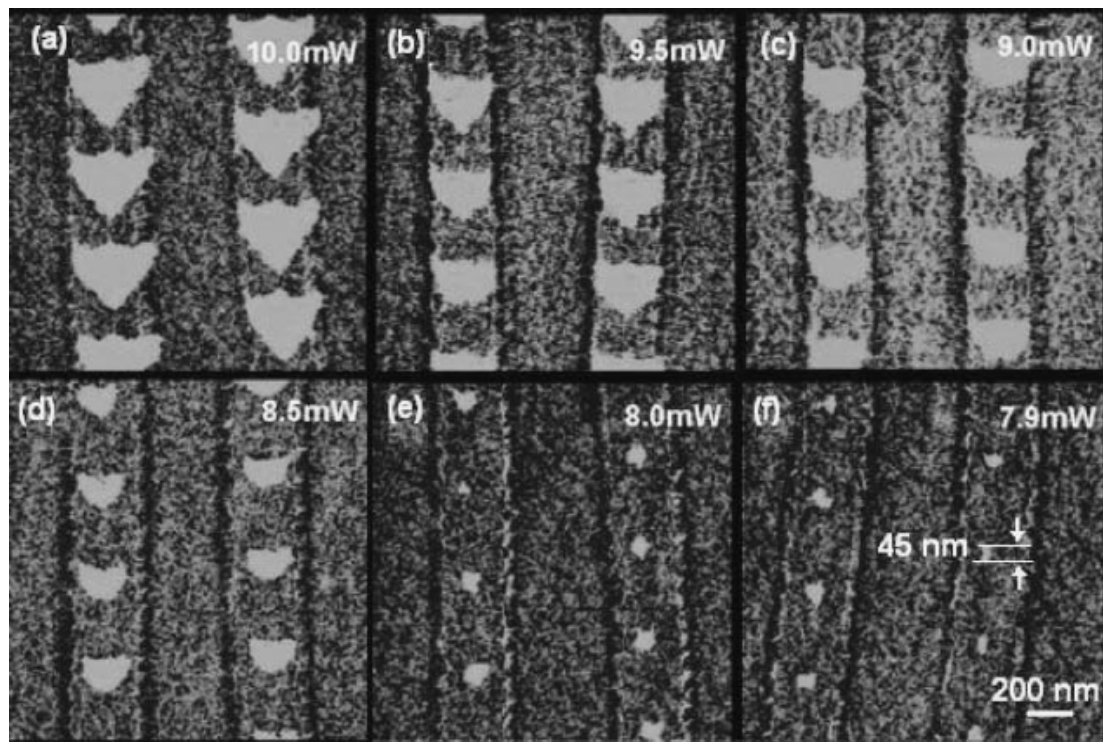

Fig. 5. C-AFM current intensity images of $1.6 \times 1.6 \mu \mathrm{m}^{2}$ scans at different laser write powers of optical disk tester (DDU-1000).

decrease in the writing time, the diameter of each recording bit declined from about 615 to $413 \mathrm{~nm}$, as shown in Fig. 4.

So far, we have successfully demonstrated that C-AFM can be used for depicting the nano scale recording bits recorded by different methods on phase-change optical disks. From these measurements, useful information on the size and shape of nano scale recording bits, and subsequently the relationship between the writing laser power and the material properties of the recording layer can be obtained.

Furthermore, we arranged several samples under a set of writing powers from 7.9 to $10.0 \mathrm{~mW}$ with the disk rotating at a constant linear velocity of $3.5 \mathrm{~m} / \mathrm{s}$, so as to understand the bit formation and the relationship between the writing power and the bit size. The results are shown in Figs. 5(a) to 5(f). The center-to-center spacing between the marks is set to be $800 \mathrm{~nm}$. Figures 5(a) to 5(f) reveal that we can make recording bits smaller in the phase-change recording layer by decreasing the writing laser power. Results show the smallest mark length to be around $20 \mathrm{~nm}$ which is the dimension of the probe we used to measure the C-AFM images. We also found a mechanism that the writing time under a constant writing power $(15 \mathrm{~mW})$ affects the recording bit size, similar to that concluded from Fig. 4. In a word, the total incident energy supplied to the phase-change recording layer is one of the main factors in the formation of nano scale recording bits on a phase-change recording layer. Using auxiliary C-AFM images of nano scale recording bits, we can study the distinct shapes of recording bits closely related to the thermal response of different types of phasechange materials.

\section{Conclusions}

We have demonstrated a brand-new, useful, and reliable method of obtaining more lively and excellent images of nano scale recording bits on commercial phase-change rewritable optical disks. Our experimental results presented herein reveal that this method is superior to other methods because of three main advantages: (1) simpler sample handling, (2) inexpensive processes, and (3) excellent contrast of signals. Moreover, the distinct C-AFM images of nano scale recording bits, including recording bits on Bluray disks and recording bits written using a static tester and commercial drivers, enable us to study the factors that affect the shape and size of recording bits in detail. In our experiment, the minimum bit size resolved in the C-AFM image is limited by the probe tip dimension and the grain 
size of the phase-change recording layer. ${ }^{14)}$ Thus, the border of nano scale recording bits can be definitely distinguished using the C-AFM method. Also together with the help of SEM or TEM, ${ }^{15)}$ a more thorough study about the properties of phase-change material and the formation mechanism of nano scale recording marks can be achieved.

\section{Acknowledgement}

The authors are grateful for the research support from the National Science Council of Taiwan, R.O.C., under project number NSC-94-2112-M-002-001 and the Ministry of Economic Affairs, R.O.C., under project number 94-EC17-A-08-S1-0006.

1) T. Ohta, K. Nishiuchi, K. Narumi, Y. Kitaoka, H. Ishibashi, N. Yamada and T. Kozaki: Jpn. J. Appl. Phys. 39 (2000) 770.

2) T. Tadokoro, T. Saiki, K. Yusu and K. Ichihara: Jpn. J. Appl. Phys. 39 (2000) 3599.

3) T. Kikukawa and H. Utsunomiya: Microsc. Microanal. 7 (2001) 363.
4) O. Ishiyama: Jpn. J. Appl. Phys. 43 (2004) 6356.

5) T. Luoh, J.-S. Bow, A. Peng, S.-Y. Tsai and M.-R. Tseng: Jpn. J. Appl. Phys. 38 (1999) 1698.

6) M. Miyamoto, A. Hirotsune, Y. Miyauchi, K. Ando, M. Terao, N. Tokusyuku and R. Tamura: IEEE J. Quantum Electron. 4 (1998) 826.

7) R. Saito, F. Ito, Y. Yokochi, T. Saito, T. Ohira, H. Sato and M. Itonaga: Jpn. J. Appl. Phys. 43 (2004) 4799.

8) J. Tominaga, T. Nakano and N. Atoda: Appl. Phys. Lett. 73 (1998) 2078.

9) T. Fukaya, J. Tominaga, T. Nakano and N. Atoda: Appl. Phys. Lett. 75 (1999) 3114.

10) T. Fukaya, D. Buechel, S. Shinbori, J. Tominaga, N. Atoda, D. P. Tsai and W. C. Lin: J. Appl. Phys. 89 (2001) 6139.

11) D. P. Tsai and W. C. Lin: Appl. Phys. Lett. 77 (2000) 1413.

12) W. C. Liu, C. Y. Wen, K. H. Chen, W. C. Lin and D. P. Tsai: Appl. Phys. Lett. 78 (2001) 685.

13) M. Mansuripur, J. K. Erwin, W. Bletscher, P. Khulbe, K. Sadeghi, X. Xun, A. Gupta and S. B. Mendes: Appl. Opt. 38 (1999) 7095.

14) B. J. Kooi, W. M. G. Groot and J. Th. M. De Hosson: J. Appl. Phys. 95 (2004) 924.

15) E. R. Meinders and M. H. R. Lankhorst: Jpn. J. Appl. Phys. 42 (2003) 809. 